\title{
MATHEMATICAL MORPHOLOGY PRE-PROCESSING FOR ENHANCED SEGMENTATION OF HETEROGENEOUS SPATIAL REGIONS.
}

\author{
Julien Radoux ${ }^{1}$, Pierre Defourny ${ }^{1}$ \\ 1: Université catholique de Louvain, Earth and Life Institute, B-1348 Louvain-la-Neuve, Belgium
}

\begin{abstract}
Résumé
La très haute résolution spatiale des images de la constellation Pléiades permet de détecter des objets géographiques de petite taille (arbres isolés, bâtiments, etc). Cependant, la délimitation de régions spatiales complexes (forêts ouvertes ou paysages urbains), est rendue plus difficile par le grand niveau de détail de l'image. En effet, les méthodes automatisées de segmentation d'image produisent souvent des résultats sur-segmentés à cause de l'hétérogénéité spectrale de ces régions. Par ailleurs, la délimitation manuelle souffre souvent d'un biais lié au photo-interprète, et même d'un manque de cohérence lorsque la même personne digitalise durant plus d'une heure. Dans cette étude, nous cherchons un nouveau filtre adapté aux images de très haute résolution spatiale afin d'améliorer la cohérence contextuelle de la segmentation par ordinateur tout en préservant la précision géométrique lors de la délimitation de régions homogènes. Le filtre proposé se base sur le principe de la morphologie mathématique, qui consiste à appliquer un ensemble de règles à une image sur base d'une forme de référence. Deux filtres composites ont ensuite été créés afin de conserver l'emplacement des limites : un filtre d'ouverture et un filtre de fermeture. Le filtre d'ouverture étend les parcelles de végétation à l'intérieur des régions spatiales hétérogènes. A l'inverse, le filtre de fermeture enlève ces parcelles isolées. Les deux filtres ont été testés sur une image Pléiades en Belgique, aux alentours de la ville de Leuven. L'image composite et l'image originale ont été segmentées avec le logiciel e-Cognition en utilisant le même jeu de paramètres. Les résultats montrent que la segmentation des images filtrées est spatialement plus cohérente que la segmentation basée sur l'image originale. La sur-segmentation est en effet réduite dans les zones hétérogènes. Par ailleurs, la précision de la délimitation des zones homogènes est améliorée significativement. Les objets issus de la segmentation de l'image filtrée sont donc plus aisément utilisables pour le suivi de régions spatiales.
\end{abstract}

Mots clés : Pléiades, Délimitation automatique, morphologie mathématique

\begin{abstract}
The very high spatial resolution of Pleiades images allows for the detection of small spatial objects such as buildings or isolated trees. However, the delineation of spatial regions, defined as associations between different spatial objects (such as open canopy forests or urban areas), becomes more challenging with the high level of details. On one hand, automated image segmentation algorithms often yield over-segmented polygons due to due to the high spectral heterogeneity of those regions. On the other hand, manual delineation was shown to end up with a significant bias from the interpreter and even a lack of consistency when the same person works more than one hour on the same task. In this study, we aimed at implementing a new filter to increase the contextual consistency of automated segmentation while preserving the geometric precision of the delineation of spectrally homogeneous spatial regions. A new mathematical morphology approach is proposed, which consists in applying a set of rules to an image based on the presence of absence of vegetation pixels within a structuring element. Two composite filters were then built based on the new filters. The opening filter removes isolated vegetation patches inside heterogeneous spatial regions, while the closing filter fills the gaps between those vegetation patches. The filters have been tested on a Pleiades images located in Belgium around the city of Leuven. A composite image was then created with the NIR and Red filtered bands stacked with the original image bands. The composite and the original bands were then segmented using e-Cognition software with the same parameters. The results show that the segmentation of the filtered images is spatially more consistent than the segmentation based on the unfiltered image. The over-segmentation is reduced in the heterogeneous areas, while the precision of the delineation is improved. The objects derived from the filtered images are thus more appropriate for the monitoring of spatial regions.
\end{abstract}

Keywords : Pléiades, Automated delineation, Mathematical morphology

\section{Introduction}

Remote sensing image analysis represents an important tool for looking at ecosystem diversity and various structural aspects of individual ecosystems (Innes, J.L. and Koch, B., 1998). Indeed, advances in the spatial and spectral resolutions of sensors now available to eco- logists are making the direct remote sensing of certain aspects of biodiversity increasingly feasible, especially for large area characterizations of biodiversity in a systematic, repeatable, and spatially exhaustive manner(Duro et al., 2007). Landscapes and ecosystems are however complex systems composed of a large number of hete- 
rogeneous components (Hay et al., 2003). As a result of this complexity, the description of biotopes will change according to the scale of observation. The analysis of the image content therefore needs to be based on an interpretation key rigorously defined by the conceptual model of the map.

Spatial region, as defined by Bian (2007), is a useful model for ecological modelling. In many cases, spatial regions indeed combine different elementary spatial objects which combine into biotopes. The thematic class of these spatial regions can be unambiguously defined based on standard classification system (Radoux and Bogaert, 2014) such as the Land Cover Classification System (LCCS) of the FAO (Di Gregorio and Jansen, 2000; Di Gregorio, 2005). Those classification systems describe the land cover based on hierarchical top down decision rules as well as the proportion of some elementary spatial objects.

With submetric spatial resolution images, such as Pléiades, spatial regions need to be delineated in order to be described and finally classified, because the pixels are smaller than their various components. Those requirements are part of a paradigm shift in digital image processing : geographic object-based image analysis (Blaschke et al., 2014), which is based on the results of image segmentation or manual delineation of image-segments.

The automated delineation of spatial regions is hindered by the high level of details in an image. Indeed, most of the image segmentation algorithms are based on the rationale that the spectral homogeneity within imagesegment is larger than between image regions. As a result, these algorithms tend to yield over-segmented results in case of heterogeneous landscape. Some advanced segmentation algorithms (VanCoillie et al., 2008) therefore include textural information in addition (or in place of) the spectral homogeneity criterion. Those are mainly efficient with regular texture (e.g. closed coniferous forests) but still missing for composite spatial region where the patterns are irregular.

The general objective of our project is to map natural and semi-natural habitats into spatial regions. The more specific aim of this study is to reduce the oversegmentation of heterogeneous spatial regions while preserving the high geospatial precision of Pléiades images. A specific pre-processing filter has therefore been developed and in combination with a hierarchical segmentation process.

\section{Data and study area}

The proposed method has been tested on a study area (figure 1) located around the city of Leuven, about $20 \mathrm{~km}$ East of Brussels (Belgium). This study area is characterized with the presence of a variety of land cover types, including built-up areas, grassland, cropland, water, broadleaved forest and needle-leaved forest, in a fragmented landscape. A large scale biological valuation map is available for the validation of the segmentation results. This map provided a very rich description of the natural biotopes based on field inventories. The legend was simplified in order to match the main land cover categories discriminated by remote sensing.

The input data was collected on August 16th 2013 by the HR sensor on board of Pléiades $1 \mathrm{~A}$ satellite. The image was composed of Near-Infrared, Red, Green and Blue bands at a native spatial resolution of 2.8 meters. It was delivered as an ortho-rectified product resampled to 2 meters. The image needed to be translated based on a ground control in order to correct a small shift due to the use of SRTM for the orthorectification. The digit numbers were then converted to top of atmosphere reflectances using Orfeo Toolbox (Christophe and Inglada, 2009).

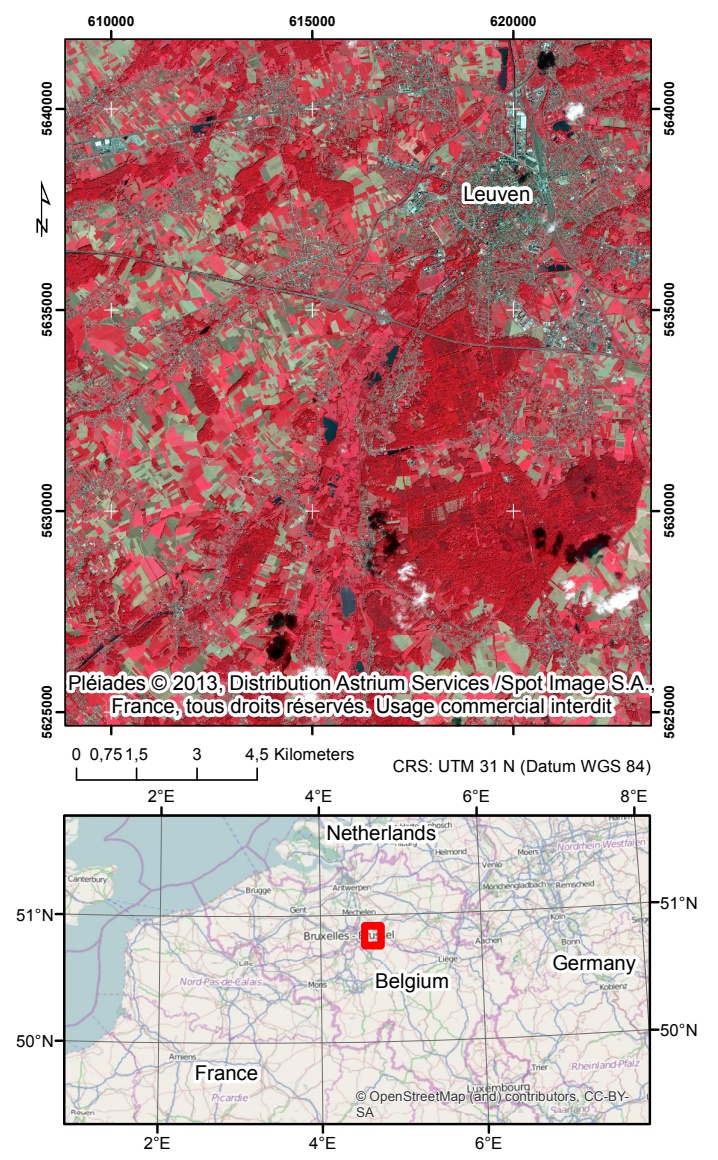

FIGURE 1: NIR-Red-Green colour composite of the input Pléiades image and location of the study area.

\section{Method}

The proposed method combines a hierarchical segmentation process and a pre-filtering. The pre-filtering reduces the structural variability of the spatial regions for subsequent segmentation. The hierarchical segmentation is used to consolidate the precision of the location of the boundaries.

\subsection{Filtering}

The filtering of the image is based on mathematical morphology. Mathematical morphology (MM) is a theory 
and technique for the analysis and processing of geometrical structures which has many application to the field of remote sensing (Soille and Pesaresi, 2002). It was initially designed for binary image analysis. The basic morphological operators are erosion, dilation, opening and closing. The core approach of binary morphology is to probe an image with a pre-defined shape, called structuring element, drawing conclusions on how this shape fits or misses the shapes in the image. The erosion of $A$ by $B$ can be understood as the locus of points reached by the center of B when B moves inside A. On the opposite, the dilation of $A$ by $B$ can be viewed as the locus of the points covered by $B$ when the center of $B$ moves inside A. Opening and closing are obtained by applying consecutively erosion then dilation (opening) or dilation then erosion with the same structuring element. As a result, elements are connected or disconnected but the location of the main edges is preserved. More details can be found in Soille (2003).

MM has been further adapted to gray scale and multispectral images. In this study, a combination of features has been added to the MM filter in order to address spatial regions that are composed of spatial objects with and without vegetation. First, the size of the structuring element is not fixed in order to preserve boundaries between different vegetation types. Second, the structuring element is tested against the NDVI using a threshold to discriminate vegetation and non vegetation.

The proposed MM filter uses an adaptive structuring element (Debayle and Pinoli, 2006a), which has been successfully used to improve the segmentation of grey scale images based on the watershed algorithm (Debayle and Pinoli, 2006b). The structural element of the proposed MM filter is a disk in order to avoid preferential directions in the results. For each pixel of the image, the radius of the disk will be incremented by a step of one pixel until strictly more than one pixel have been identified as vegetation or the maximum radius is reached. The aim of the incrementation is to preserve the detailed spectral information in areas that are not heterogeneous according to the vegetation criteria.

The selection of vegetation pixels is performed based on a threshold on the NDVI. Furthermore, dark pixels are ignored because they are likely to belong to shadows or water, and shadows are the main sources of heterogeneity in closed-canopy forests. If the maximum radius is reached without covering any vegetation pixels, the pixel value of the central pixel is copied to the filtered image. Otherwise, the spectral values of the pixel with the maximum NDVI value is considered. By copying all the spectral signature of the pixel of maximum NDVI, the final spectral signatures are consistent with those captured by the sensor. This would not be the case if each spectral band was processed independently.

For the erosion filter, the radius is incremented until more than one pixel have been identified as non vegetated, and the spectral values of the pixel with the minimum NDVI value is used. Opening (and closing) are obtained by successively applying erosion and dilation (dilation and erosion) on the image with the same parameters.

The parameters have been selected according to a preliminary analysis of the image. A maximum radius of 20 pixels has been selected based on the maximum distance between houses in the sparse urban areas. For the opening, the minimum NDVI value of a sample of vegetation pixels was selected as a NDVI threshold, and the maximum value of a sample of non vegetation pixels was used for the closing. Those value equalled 0.2 and 0.5 respectively for closing and opening. The use of extreme values helps to avoid the dilation of mixed pixels and hence increase the contrast between vegetated and non vegetated regions.

\subsection{Segmentation}

In this study, the multi-resolution segmentation algorithm (Baatz and Schäpe, 2000) was selected for the segmentation of the images. It consists in a hierarchical segmentation algorithm based on the region growing strategy. It is widely used in the literature and considered as one of the best methods (NM2003), but it requires some tuning based on several parameters. The image-objects are iteratively merged with the neighbours that will result in the smallest value of a post merging index. The iteration stops when this index is larger than the predefined scale parameter. The index has a spectral and a shape component that are combined in a weighted sum according to the shape parameter. The spectral component is computed as a weighted sum of the variance of the different spectral bands. The shape constraint is used to avoid complex shapes by alleviating strong spectral constraints. It is composed of a weighted sum of smoothness and compactness indices, which minimize the irregularities of the contour (smoothness) or the ratio between the square of the perimeter and the area (compactness).

The image-objects were created in two steps (figure 2) based on a stack of the original image with the images resulting from both MM filters. Different sets of parameters have been selected in order to account for the multiscale structure of the image. A first level of segmentation was used to identify spatial objects. This level used a small scale parameter and gave the prevalence to the spectral information of the original (non filtered) image (scale $=30$, shape $=0.1$, compactness $=1)$. Because of the small scale parameter and the use of the original image, the first level is over-segmented, but it preserves the boundaries. The second level takes advantage of the hierarchical algorithm by merging the spatial objects into spatial regions (scale $=200$, shape $=0.3$, compactness $=1$ ). The larger scale parameter and the use of the filtered image information indeed reduces the over-segmentation. At this step, the NIR and Red bands of the opened and closed images dominate the spectral information, and a larger shape parameter is used to favour the creation of compact image-segments. Because of the use of the first level, the rounding artefacts at the 
(A) Small image-object on top of the original image

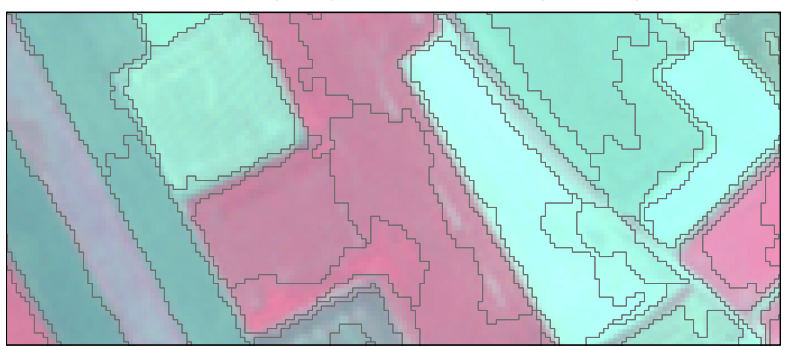

(B) Final segmentation result on top of the opened image

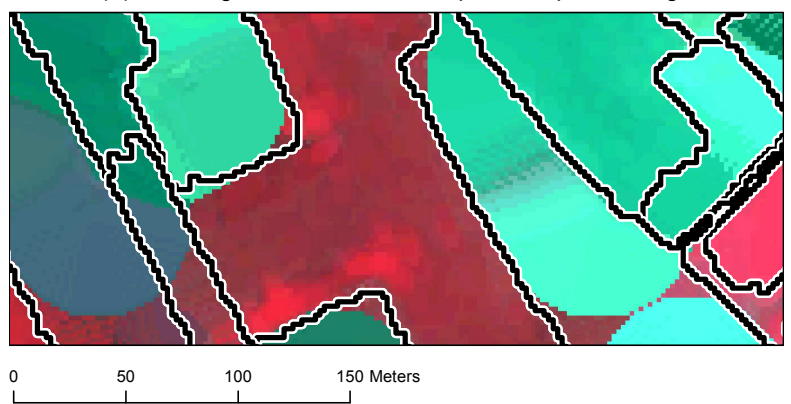

FIGURE 2: Hierarchical segmentation used to avoid the rounded corners resulting from the morphological filters. On top (A), small objects create based on the original spectral bands. At the bottom (B), final segmentation results obtained by merging small object based on the filtered bands. The background images are NIR-Red-Green composites.

corners of the spatial regions are avoided in the final result.

For the sake of comparison, the image was also segmented with the same set of parameter, but without the spectral bands resulting from the morphological filters. In this second segmentation, the weight of each original band is set to the sum of the weights of the corresponding spectral bands used in the other segmentation, so that each spectral band has the same contribution in both cases.

\subsection{Quality assessment}

There is no standard method for the accuracy assessment of delineation results, both because geographic object-based image analysis is a new paradigm and because their are different objective behind image segmentation. In this study, the aim is to achieve the delineation of functionally consistent landscape units. Overand under-segmentation indices have therefore been selected. They are measured with respect to the biological valuation map using the generalization index proposed by Carleer et al. (2005), which is the ratio between the number of image-segments and the number of polygons in the reference map.

In addition, the precision of the delineation along the boundaries is measured using the mean and variance of the distance between reference and the segmentation results (Radoux and Defourny, 2007). A total of 196 points were distributed based on random seeds on three types of boundaries, namely crop/crop, crop/forest and crop/urban. From those points, 20 have been discarded because the map did not match the boundaries observed on the image at these locations. The delineation quality indices were therefore derived from the distance between 176 points and the closest boundaries of the same type as those points in the reference dataset.

\section{Results}

The results of the MM filters helped to highlight two types of spatial regions. On one side, the homogeneous regions are little affected by the filter and have an unchanged spectral signature. On the other side, the heterogeneous regions appear either fully vegetated or fully non vegetated on the filtered images. For instance, two types of urban areas are highlighted by the closing of the image : the city center that is completely impervious, and the rest of the city that includes urban greens (figure 3). In the forested areas, the opening also allows to identified gaps with a larger contrast. The structural information of the image is thus well highlighted.

At a smaller scale, the precision of the long edges is well preserved by the two filters. Furthermore, the shadows from trees are removed by the closing filter, which is another useful feature for the subsequent delineation. However, the corners have been rounded because of the shape of the structural element. Compared with the MM filters with a fixed-size structuring element, the use of an adaptive structuring element preserved more details in the resulting images (figure 4).

The visual assessment of the segmentation results shows there is no loss of precision along the detected boundaries compared with the original image. For the crop/crop and the crop/forest boundaries, the bias and the standard deviation of the edge location were not significantly different. In both cases, the bias was smaller than a third of a pixel. The standard deviation of the crop/crop boundaries was respectively 4.0 and 4.1 meter for the segmentation of the original and the filtered image. The standard deviations were larger in the case of the crop/forest boundaries, with respectively 7.1 and 7.4 meters. The case of the crop/urban boundaries was more problematic. There were indeed large discrepancies with the reference data (up to $50 \mathrm{~m}$ ) that induced very large standard deviation. Even after the removal of outliers, the standard deviation was of 8.4 meters for the results based on the original image and 9.8 for the filtered image.

The results show that the segmentation of the filtered images is spatially more consistent than the segmentation based on the unfiltered image. The use of identical segmentation parameters yielded a similar number of image-segments. There were 4 percent less polygons in the case of the filtered image. The number and the delineation precision of crop parcels was very similar for both cases. The main difference were, as expected, for the most heterogeneous areas (open natural biotopes and low density urban areas). In this case, there were 40 percent less polygons when the segmentation was applied on the filtered image. In the slightly heterogeneous 
(A) Closing

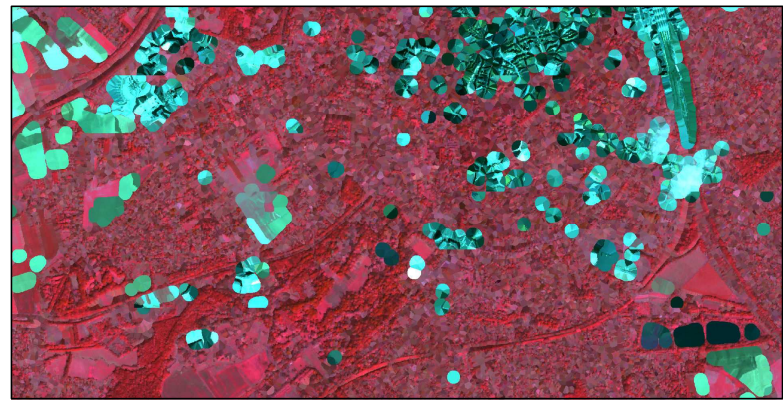

(C) Original

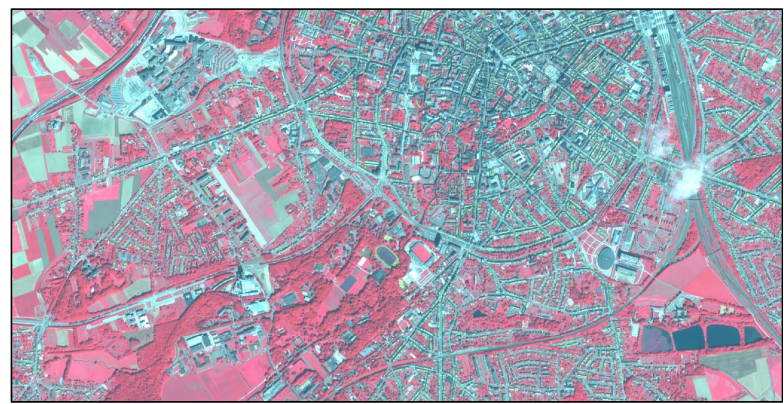

(C) Opening

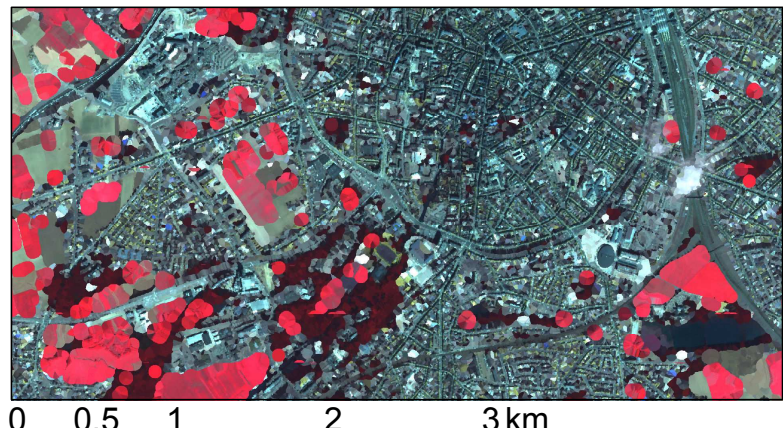

$\begin{array}{lllll}0 & 0,5 & 1 & 2 & 3 \mathrm{~km}\end{array}$

FIGURE 3: Results of the new mathematical morphology filters on a Pléiades image $(A)$ closing and $(C)$ opening compared with the original image. Homogeneous areas such as city center and crop fields are not modified, while heterogeneous areas are markedly affected by the filter.

land cover at this scale, closed canopy forests and dense urban areas, the results are again quite similar. In those case, contrary to the other land cover classes, the level of over-segmentation is higher with the pre-processed image. However, the smaller size of the image-object in forested areas seems to be justified by the discrimination between broadleaved and needleleaved stands. Without preprocessing, several needle-leaved stands are indeed under-segmented.

\section{Discussion and conclusion}

This study showed that it is possible to improve the segmentation results for heterogeneous spatial regions without affecting the delineation precision. MM filters indeed yield sharp gradients between spatial regions, and the boundaries between adjacent vegetated regions are not modified thanks to the adaptive structuring element.

(A) Fixed structuring element

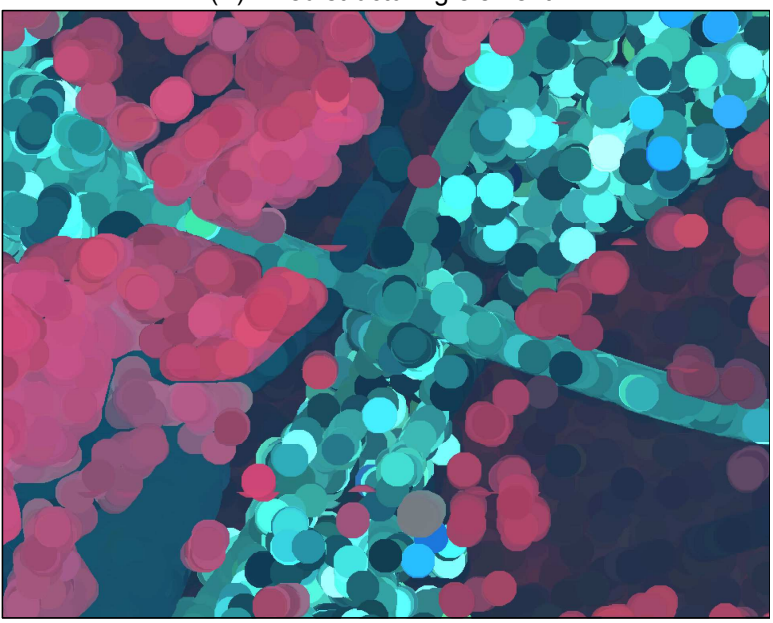

(B) Adaptive structuring element

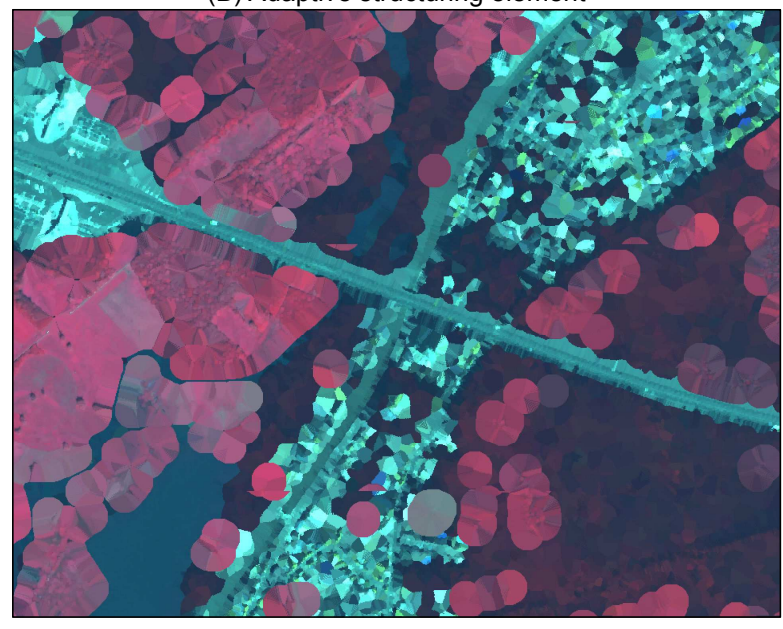

0100200300 Meters

FIGURE 4: Comparison of two opening of the same image, with (A) and without $(B)$ adaptive structuring element.

(A) Filtered

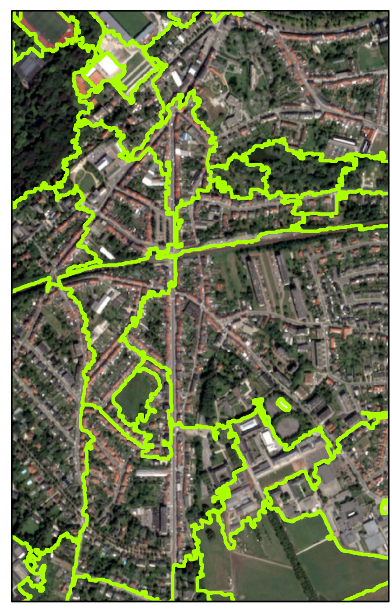

$0 \quad 250 \quad 500 \quad 750$ m

FIGURE 5: Segmentation results illustrated for the urban area with $(A)$ and without $(B)$ preliminary filtering.

The combination of the MM filter with a hierarchical segmentation algorithm remains however necessary to re- 
move the rounding artefacts at the corners.

The proposed method was based on an arbitrary selected maximum size of the structuring element, which confines the analysis to a single scale. However, though the choice of the radius can be discussed, the method helped to identify spatial structure matching the definitions of spatial regions. Furthermore, forcing the scale helps to improve the repeatability of the delineation. Indeed, even if large discrepancies have been observed with the reference dataset, the errors can be consistently explained and documented, which is a key feature in case of monitoring.

The generalisation of the method has not been quantitatively assessed. Additional results of the opening and closing filters were visually consistent on Pléiades images from other parts of Belgium. However, the parameters of the filters should probably be adjusted in the case of markedly different landscapes and/or spatial resolution of the image.

In addition to the improvement of the delineation, the image filtering also provided additional bands that can be used for the classification. Previous studies have used increasing sizes of structural elements on this purpose. In our case, there is only one scale, which is fixed, but the combined information from the opening and the closing provides meaningful contextual information for the classification of the spatial regions.

The full potential of mathematical morphology could be better exploited for remote sensing data analysis. Further work is necessary to automatically adjust the reflectance thresholds to the information content of the image. A standard method for the accuracy assessment of segmentation results would be a first step in this direction.

\section{Acknowledgements}

The authors would like to thank the anonymous reviewers for their valuable comments. This research was funded by the Fédération Wallonie Bruxelles in the frame of the Lifewatch-WB project. The images were kindly provided by the Pleiades RTU and PUG programs, with additional images from the Belgian Science Policy. The reference dataset came from INBO. The image filter has been implemented using the Orfeo Toolbox Library. The authors would like to thank the OTB team for their support.

\section{Références}

Baatz, M., Schäpe, A., 2000. Multiresolution segmentation - an optimization approach for high quality multi-scale image segmentation. In : Strobl, J., Blaschke, T., Griesebner, G. (Eds.), Angewandte Geographische Informationsverarbeitung XII. Wichmann-Verlag, Heidelberg, pp. 12-23.

Bian, L., JUN 2007. Object-oriented representation of environmental phenomena : Is everything best represented as an object? Annals of the Association of American Geographers 97 (2), 267-281.

Blaschke, T., Hay, G., Kelly, M., Lang, S., Hofmann, P., Addink, E., Queiroz Feitosa, R., van der Meer, F., van der Werff, H., van Coillie, F., Tiede, D., 2014. Geographic Object-Based Image Analysis - Towards a new paradigm. ISPRS Journal of Photogrammetry and Remote Sensing 87, 180-191.

Carleer, A., Debeir, O., Wolff, E., 2005. Assessment of very high resolution satellite image segmentations. Photogrammetric Engineering and Remote Sensing 71 (11), 1284-1294.

Christophe, E., Inglada, J., 2009. Open source remote sensing : Increasing the usability of cutting-edge algorithms. IEEE Geoscience and Remote Sensing Newsletter 150, 915.

Debayle, J., Pinoli, J.-C., 2006a. General adaptive neighborhood image processing - part I. Journal of mathematical imaging and vision 25 (2), 245-266.

Debayle, J., Pinoli, J.-C., 2006b. General adaptive neighborhood image processing - part II. Journal of mathematical imaging and vision 25 (2), 267-284.

Duro, D. C., Coops, N. C., Wulder, M. A., Han, T., 2007. Development of a large area biodiversity monitoring system driven by remote sensing. Progress in Physical Geography 31 (3), 235-260.

Hay, G. J., Blaschke, T., Marceau, D. J., Bouchard, A., 2003. A comparison of three image-object methods for the multiscale analysis of landscape structure. ISPRS Journal of Photogrammetry and Remote Sensing 57 (5), 327-345, challenges in Geospatial Analysis and Visualization.

Innes, J.L. and Koch, B., 1998. Forest Biodiversity and Its Assessment by Remote Sensing. Global Ecology and Biogeography Letters 7 (6), 397-419.

Di Gregorio, A., 2005. Land cover classification system : Classification concepts and user manual for software version 2 . FAO Environment and Natural Resources Service.

Di Gregorio, A., Jansen, L., 2000. Land cover classification system (Iccs) : Classification concepts and user manual. GCP/RAF/287/ITA Africover-East Africa Project and Soil Resources, Management and Conservation Service, Food and Agriculture Organization.

Radoux, J., Bogaert, P., 2014. Accounting for the area of polygon sampling units for the prediction of primary accuracy assessment indices. Remote Sensing of Environment 142, 9-19.

Radoux, J., Defourny, P., 2007. A quantitative assessment of boundaries in automated forest stand delineation using very high resolution imagery. Remote Sensing of Environment 110, 468-475

Soille, P., 2003. Morphological Image Analysis : Principles and Applications, 2 Edition. Springer-Verlag New York, Inc., Secaucus, NJ, USA.

Soille, P., Pesaresi, M., Sep 2002. Advances in mathematical morphology applied to geoscience and remote sensing. Geoscience and Remote Sensing, IEEE Transactions on 40 (9), 2042-2055.

VanCoillie, F., Verbeke, L., DeWulf, R., 2008. Semi-automated forest stand delineation using wavelet based segmentation of very high resolution optical imagery. In : Blaschke, T., Lang, S., Hay, G. (Eds.), Object-Based Image Analysis. Lecture Notes in Geoinformation and Cartography. Springer Berlin Heidelberg, pp. 237-256. 